\title{
América Latina, el gran latifundio mediático
}

\section{Andrés Mora Ramírez ${ }^{56}$}

En su prólogo al libro Periodistas y magnates, Armand Mattelart (2006) afirma que, después de la caída del Muro de Berlín y con la irrupción de las nuevas tecnologías de la información, se ha puesto en marcha, a escala global, un "proyecto de sociedad" determinado por el uso y control del recurso informacional.

¿Qué características tendría esa sociedad, cuando asistimos hoy al fenómeno de la concentración de la propiedad de los medios de comunicación -y de las industrias culturales- en unos cuantos conglomerados empresariales? ¿Qué lugar ocuparian y qué funciones desempeñarían los países latinoamericanos?

En el apogeo de la globalización neoliberal, América Latina, esa "inmensa provincia del subdesarrollo", al decir del escritor uruguayo Mario Benedetti (1987), se asemeja cada vez más a un gran latifundio mediático, dominado por

\footnotetext{
56 Periodista. Magister en Estudios Latinoamericanos de la Universidad Nacional de Costa Rica.
} 
los poderosos grupos transnacionales de la comunicación y sus interlocutores regionales.

Los datos son contundentes: Estados Unidos y la Unión Europea controlan el $90 \%$ de toda la información del planeta; de las 300 principales compañías del sector, 144 son de Estados Unidos, 80 de la Unión Europea y 49 de Japón. En contraste, los países pobres, donde vive el $75 \%$ de la humanidad, poseen únicamente el $30 \%$ de los periódicos del mundo (Uribe, 2005).

Un estudio reciente del Instituto Prensa y Sociedad (Mastrini y Becerra, 2006) sobre la concentración de medios, efectuado en nueve países de América Latina, determinó que "el primer operador acapara, en promedio, más del $30 \%$ del mercado, mientras que los cuatro primeros superan el $80 \%$. El medio con mayor índice de concentración es la TV abierta, con $85 \%$, seguido por la TV por cable $(84 \%)$ y la prensa $(62 \%)$. La radio es el medio menos concentrado, con $31 \%$ de cuota de mercado para los cuatro primeros operadores". Entre esos primeros operadores figuran empresas de los grupos: Televisa de México, Cisneros de Venezuela, Globo de Brasil y Clarín de Argentina.

Distintos autores coinciden en que los altos índices de concentración de la propiedad - uno de los mecanismos de control del recurso informacional- comprometen seriamente la realización de los supuestos que dan sustento a un sistema democrático. El proceso en curso, afirman Mattelart (2003) y Ramonet (2001), amenaza la diversidad cultural, pone en desventaja a los países y grupos de población más pobres y limita la diversidad de fuentes para que los ciudadanos puedan conocer y participar de los asuntos públicos.

Esta situación refleja en forma dramática la agudización de las desigualdades económicas, culturales y tecnológicas que coartan las posibilidades de desarrollo de los países latinoamericanos, y del Tercer Mundo en general, como 
viene siendo denunciado por organismos internacionales desde hace casi tres décadas.

En efecto, en 1980 el Informe de la Comisión Internacional sobre Problemas de la Comunicación, conocido como Informe Macbride, advirtió al foro de la Conferencia General de la UNESCO que "la industria de la comunicación está dominada por un número relativamente pequeño de empresas que engloban todos los aspectos de la producción y la distribución, las cuales están situadas en los principales países desarrollados y cuyas actividades son transnacionales" (UNESCO, 1980).

En 1995, una vez más la UNESCO, en su informe Medios de Comunicación y la Democracia en América Latina y el Caribe, concluyó que "concentración significa posesión de los medios en menos manos, y ello estrecha la pluralidad que se promueve en el campo político y dificulta el florecimiento democrático (...)".

En uno de los trabajos incluidos en este documento, Luis Suárez, entonces Secretario General de la Federación Latinoamericana de Periodistas, explicaba que las tendencias a la desregulación, el libre mercado, y el abandono por parte de los Estados de actividades esenciales como la comunicación y la cultura, asumidas ahora por agentes privados, dificultaban la democratización de las sociedades de la región.

Las paradojas de la globalización estaban en el centro de este problema: "al mismo tiempo que se proclama y ejerce la libertad de mercado, esa misma posibilidad, con la ausencia del compromiso estatal y la debilidad económica de las organizaciones sociales, fortalece la monopolización y concentración, incluso extranjera, de los medios de comunicación". (UNESCO, 1995).

Más recientemente, la Relatoría de la Libertad de Expresión de la Organización de Estados Americanos (OEA), en su informe anual del 2004, dio cuenta de "las 
continuas denuncias recibidas (...) en relación con prácticas monopólicas y oligopólicas en la propiedad de los medios de comunicación social", así como de la preocupación de distintos sectores de la sociedad civil por lo que esto puede representar para garantizar el pluralismo político e informativo.

Para la Relatoría, la existencia de estas prácticas "afecta seriamente la libertad de expresión y el derecho de información de los ciudadanos de los Estados miembros, y no son compatibles con el ejercicio del derecho a la libertad de expresión en una sociedad democrática" (OEA 2004).

La afectación, en mayor o menor medida, de Derechos Humanos y Culturales fundamentales, dice de un sistema de medios de comunicación que, emulando la estructura de propiedad de los latifundios del siglo XIX y principios del XX, genera un tipo de relaciones sociales e intercambios simbólicos basados en el autoritarismo y la subordinación al poder del más fuerte.

Si José Saramago (2003), el Premio Nobel de Literatura, describía en una de sus novelas al latifundio como “...este presente de tierra ahora repartida entre los dueños del hacha y según el tamaño y el hierro o filo del hacha...", en el caso de los medios en América Latina sería preciso hablar de un espacio audiovisual e impreso distribuido según el poder financiero y los intereses de los actores privados, y no con arreglo a los derechos de los ciudadanos y ciudadanas.

Universalmente se reconoce y destaca la importancia de los medios en la integración social, en la aplicación de políticas culturales e incluso en la reafirmación de la identidad y los modos de expresión de millones de personas (UNESCO, 1980). De ahí que, por sus profundas y complejas implicaciones, la concentración de la propiedad sea un problema directamente vinculado con las transformaciones y el futuro de la cultura, la calidad de la democracia y la memoria colectiva de los pueblos latinoamericanos. 
Por esta misma razón, resulta casi imposible no expresar el desasosiego que produce el panorama actual de la región, especialmente ante "la posibilidad que todo ese inmenso campo de la información sea manejado por cuatro o cinco grupos de poder, tan ajenos a las reales necesidades e inquietudes de la sociedad y tan alejados de sus intereses y ansiedades, como lo están los satélites que dan vuelta por el espacio y desde allí dominan o pretenden dominar las comunicaciones" (Arbila, 2006).

\section{La globalización y la industria de los medios de comunicación}

El proyecto de sociedad que avizora y sobre el que advierte Mattelart, es el proyecto de sociedad de la globalización neoliberal, que coloca a la rentabilidad económica y la competitividad como incontestables criterios de validez.

Se trata, como explica Castells (2004), de un sistema global constituido a partir de redes de intercambio y flujos de comunicación, que "es a la vez extremadamente incluyente y extremadamente excluyente. Incluyente de todo lo que tiene algún valor según los códigos dominantes en los flujos, y excluyente de todo aquello que, según dichos códigos, no tiene valor o deja de tenerlo".

En América Latina, los procesos económicos, sociales, políticos y culturales que hacen a la globalización, se expresan con mayor intensidad a partir de los años 1980 y 1990, con la aplicación indiscriminada, en prácticamente todos los países, de los programas de reforma y ajuste estructural inspirados en el marco ideológico del Consenso de Washington.

Estas políticas fueron orientadas hacia (y en ocasiones forzaron) la apertura de mercados, la liberalización de los flujos financieros y la privatización de activos estatales. 
Mattelart (2006) considera que la inflexión del orden jurídico hacia las leyes del mercado, consumada en 1998 con el Acuerdo sobre Liberalización de las Telecomunicaciones en la Organización Mundial del Comercio, creó las condiciones para el desarrollo de una estructura oligopólica de la propiedad de los medios de comunicación, reclutando "sus primeros adeptos en los regímenes neoliberales como en el caso de (...) Argentina, Chile, México o Venezuela".

En este período se manifestaron dos características fundamentales del proyecto de sociedad de la globalización neoliberal: la concentración de la riqueza (incluidos los factores de producción comunicacionales) y el aumento de la desigualdad. Por un lado, la iniciativa privada se expandió en la región y numerosas empresas públicas de medios de comunicación y telecomunicaciones fueron subastadas, en polémicas privatizaciones, entre grupos interesados en aumentar su poder político y financiero; y por el otro, la economía latinoamericana se estancó y puso al descubierto la inequidad en la distribución de la riqueza generada por las políticas neoliberales, evidente en el hecho de que, según los datos de CEPAL, para el año 2007, el 34\% de la población latinoamericana todavía se encontraba en condición de pobreza.

La imagen del latifundio mediático -que también se extiende a otros ámbitos- se refuerza en la misma medida en que se constatan las escandalosas contradicciones entre los emergentes ricos latinoamericanos (grupos de empresas familiares de la región y grupos transnacionales) y los cada vez más numerosos pobres, que estarán culturalmente subordinados a los propietarios y detentadores del recurso informacional.

Así, en el proyecto de sociedad de la globalización neoliberal, el sistema de los medios desempeña una función de organización del mundo simbólico y económico, a través de procesos de inclusión/exclusión, configurando 
"nuevas relaciones de fuerza entre economías, entre culturas, entre Estados, nuevas formas de hegemonía, modos inéditos de gobernanza de las sociedades contemporáneas y del planeta" (Mattelart, 2006).

En lo que puede definirse como la profundización de un paradigma comunicacional autoritario, las empresas propietarias de medios tienden a concentrar o fusionar sus actividades, para mantener y asegurar la posición dominante en el mercado. En adelante, será el poder financiero, y no los valores culturales o democráticos, los que impondrán las pautas de la comunicación social.

De esta forma, sostiene Guinsburg (2001), queda "relegada la esencia de la educación, el entretenimiento y la información por la conversión del ciudadano en consumidor. La política, por su parte, queda relegada a la oferta publicitaria. Esta fenomenología se centra en la cruenta ocupación del espacio de la comunicación social por grupos económicos de actividad diversificada en negocios de diferente índole".

Mastrini y Becerra (2006) analizan, precisamente, la estructura de este sistema y las interrelaciones comerciales entre sus protagonistas, estableciendo tres niveles de participación:

- En el primer nivel, se ubican los principales grupos transnacionales, como General Electric, AT\&T, Disney, Time Warner, Sony, News Corp., Viacom, Seagram y Bertelsmann. A partir del mercado estadounidense, estas compañías han desarrollado redes globales que les permiten operar "con todo el planeta como mercado y cuyas cotas de penetración en las diferentes regiones y países encuentran pocas barreras".

- En el segundo nivel, se agrupan a cerca de 50 grandes grupos con sede en Europa, Estados Unidos o Japón, 
cuyas operaciones se verifican a escala regional (entre varios estados). Entre estos se encuentran: Dow Jones, Comcast, The New York Times, The Washington Post, Hearst, McGraw Hill, CBS, Times-Mirror, Reader's Digest, Pearson, Kirch, Havas, Mediaset, Hachette, Canalt, Prisa y Reuters. Los autores consideran que dichos grupos son "el núcleo dinámico del sistema global, pues establecen relaciones con los aproximadamente diez primeros, que se encuentran en posición dominante, y permiten traducir las estrategias de los más grandes a los entornos regionales más apetecibles como nichos de mercado, pues constituyen áreas geográficas centrales por los ingresos de los consumidores allí radicados".

- El tercer nivel está conformado por unas 90 corporaciones líderes de mercados subregionales y regionales, entre las que se cuentan las más importantes empresas latinoamericanas. Mastrini y Becerra señalan que "estos actores poderosos en la región antes estaban más supeditados a las tradiciones y condiciones locales y nacionales que ahora", cuando sus vínculos con las transnacionales son más estrechos y, sobre todo, condicionados desde una perspectiva financiera.

Esta última observación remite a uno de los debates más polémicos en el ámbito de los estudios de la comunicación y la cultura, y de las relaciones entre los llamados Primer y Tercer Mundo: la acción de las empresas transnacionales no solo como movilizadoras de capitales y tecnologías, sino como agentes capaces de "modificar la orientación sociocultural de toda la sociedad" (UNESCO, 1980), dada su enorme influencia sobre los aparatos de producción económica y simbólica. 


\section{Distribución de la propiedad de los medios en América}

Latina

En el latifundio mediático latinoamericano, los altos índices de concentración hallados en las investigaciones más recientes, más allá de dar cuenta del nuevo orden de la información propiciado por las reformas neoliberales, expresan también la consolidación de un proceso histórico de identificación y asociación de los medios con los sectores y grupos hegemónicos de la región.

A diferencia del modelo que promovió el Informe Macbride, que abogaba por "el establecimiento de un nuevo sistema [de comunicaciones] basado en el principio de igualdad de derechos, la independencia y el libre desarrollo de los pueblos" (UNESCO, 1980), como condición para proteger la diversidad cultural y democratizar los flujos mundiales de información; el sistema global vigente, sobre la base de la desregulación, debilita la tutela del Estado y fortalece el poder del mercado y los actores privados, que asumen el control de la propiedad de los medios y las nuevas tecnologías (Herman y McChesney, 1999; y Mattelart, 2003).

Autoritarismo y subordinación, primacía del interés comercial sobre el servicio público, negación de las posibilidades de democratización: son todas relaciones características de la vida en el latifundio, también presentes en el actual modelo neoliberal competitivo de la comunicación, y que recorren transversalmente la historia del desarrollo de los medios y sus formas de propiedad en América Latina (Sánchez Ruiz, 1994).

\subsection{Un balance histórico de la concentración}

La biografía de los problemas vinculados a la concentración de los medios puede ser narrada desde 
dos dimensiones: una geográfica, como se observó en la década de 1960, donde la centralización de la producción, distribución y consumo cultural en los grandes centros urbanos, provocó la marginación de extensos territorios, en cuanto al acceso a la televisión, la radio, la prensa escrita y los circuitos cinematográficos. Mastrini y Becerra (2006) concluyen que, en esta situación, "la diversidad cultural de los países de la región se vio reducida en muchos casos a la visión de las elites capitalinas (...) La propiedad de los medios de comunicación en manos de los grupos hegemónicos dificultaba la aparición en los medios de voces que cuestionaran las estructuras sociales vigentes".

Los problemas asociados a la dimensión económica son más visibles a partir de los años 1990, cuando la internacionalización de los mercados prepara el terreno para la conformación de grupos de comunicación hegemónicos a escala regional, como los ya citados Televisa, Cisneros, Globo y Clarín, donde la expresión de las voces disidentes serán aún más difícil.

Estos grupos responderán a una lógica idéntica a la de los consorcios globales: la convergencia de actividades comunicacionales (telecomunicaciones, informática, industria gráfica), la participación en otras áreas de la industria y el comercio, y la intensificación de economías de escala. Pero, además, establecerán negocios cruzados entre sí y con los principales grupos a nivel mundial.

Tales alianzas, en criterio de Mastrini y Becerra (2003), proveen beneficios a ambas partes: los grupos regionales fortalecen su capacidad financiera, e incorporan nuevos contenidos y tecnología; en tanto que los grandes consorcios planetarios reducen el riesgo de inversión en nuevos mercados, aprovechando la capacidad instalada y "los contactos y la influencia política, en el sentido más amplio, que los grupos locales y regionales han venido desarrollando históricamente en su contexto". 
Sin embargo, en este intercambio, el sistema de medios global encuentra interlocutores que, como las principales empresas latinoamericanas, "complementan y vigorizan la estructura de dominancia ejercida por los principales grupos del planeta" (Mastrini y Becerra, 2003).

\subsection{Los terratenientes de la comunicación}

Las investigaciones de Mastrini y Becerra (2003 y 2006) constituyen uno de los más completos y minuciosos trabajos de análisis sobre la concentración de los medios en América Latina. Sus hallazgos permiten trazar el retrato de la realidad de la región, en términos del control de la comunicación social por unos cuantos, pero poderosos, grupos y sus empresas subsidiarias.

Una breve caracterización de estos conglomerados, a partir de los datos recabados por ambos autores, comprueba la gama de negocios que han desarrollado, los sectores estratégicos en que incursionan y el tipo de relación que mantienen con empresas similares, a nivel mundial y regional:

- Televisa (México): dispone de más de 300 estaciones de televisión en México, de las que recibe el 60\% de sus ingresos. Tiene participación accionaria en la cadena de televisión hispana Univisión, una de las más destacadas en los Estados Unidos. Además, controla el 51\% de la empresa Cablevisión, el segundo operador de televisión por cable del país, y el $60 \%$ de Innova, controladora de la señal de DTH Sky. Mantiene acuerdos comerciales con el empresario Carlos Slim, el hombre más rico de América Latina y propietario de la telefonía básica mexicana Telmex.

- Grupo Cisneros (Venezuela): pertenece à un holding industrial con múltiples inversiones (no solo 
en industrias culturales), y con ingresos anuales de más de $\$ 4.000$ millones de dólares. Se ha expandido en el control de estaciones de televisión en América Latina, con participación en estaciones en Chilevisión (Chile), Caracol (Colombia), la Caribean Communication Company (Caribe) y Venevisión (Venezuela). Estas operaciones son financiadas mediante el fondo de inversión Ibero-American Media Partners, junto al fondo financiero norteamericano Hicks, Muse, Tate \& Furst. El Grupo Cisneros es el mayor accionista de la cadena hispana Univisión y de Galavisión, ambas en Estados Unidos. Es socio de la televisión prepago DirecTV Latin America. Con la estadounidense America On Line, desarrolla AOL Latin América, uno de los principales proveedores de Internet de la región latinoamericana.

- Globo (Brasil): este grupo produce contenidos televisivos para el consumo del mercado local y para la exportación al resto del mundo. Es propietario de la principal red brasileña de TV por cable, Globocabo (Microsoft es propietaria del 15\%); del diario O'Globo, y participa en la operadora de televisión satelital SkyLA. Es propietario de compañías de radio, comunicaciones satelitales y de telecomunicaciones. Desarrolla proyectos para dar acceso a Internet a través de la TV por cable, mediante sus 25 mil kilómetros de tendido de red.

- Grupo Clarín (Argentina): editor del diario El Clarín y propietaria de Radio Mitre, una de las de mayor audiencia de Buenos Aires. En el negocio de la televisión, es dueño de Canal 13 y de la empresa de TV por cable Multicanal (con más de 1,5 millones de abonados). Incursionó en el negocio de las telecomunicaciones con la Compañía de Teléfonos del Interior 
(CTI), y en la televisión satelital a través de DirecTV Latin America. El 18\% de las acciones del Grupo Clarín pertenecen al banco estadounidense Goldman Sachs.

Pese a la aparente fortaleza de estos grupos, y la solidez de sus actividades comerciales, en los últimos años los grupos Clarín y Globo se han reunido con sus acreedores, para buscar soluciones a sus problemas financieros. Mastrini y Becerra (2006) señalan aquí otra de las paradojas de la economía globalizada: "para poder insertarse en el mercado mundial, estos poderosos actores debieron asumir importantes deudas, que en el presente les resulta muy dificultoso saldar. Sin embargo, todos los empresarios señalan que no tenían otra alternativa que encarar el proceso de crecimiento para no verse absorbidos por grupos internacionales más grandes".

Es decir, el latifundio mediático de América Latina se inserta subordinadamente dentro de un latifundio de mayores proporciones. Concentración y dependencia, como tantas otras veces en la historia del continente, se encuentran aquí también para dibujar otra faceta de la globalización.

\section{Amenazas para la diversidad cultural}

Para el caso específico de la cultura en América Latina, la UNESCO (1995) ha descrito con exactitud lo que representa la relación desigual entre nuestros países y el poder de las transnacionales de la comunicación: "una globalización absorbente (...) desplaza de la actividad a empresarios y editores nacionales apegados cuando menos a las tradiciones culturales de sus respectivos países, con amor a la lengua y a las ideas de la integración latinoamericana y del iberoamericanismo. Y como globalidad y concentración 
significan también engarce y desposesión por parte de los grandes centros y sedes monopólicos, en buena medida el fenómeno puede conducir a una cierta, y en algunos casos segura, desnacionalización".

El desplazamiento no obedece únicamente a las asimetrías económicas que, en un esquema de libre mercado, colocan en desventaja a los más débiles. Los intereses de los defensores del status quo comunicacional (reflejo de un orden político, social y económico), conspiran contra los empeños -individuales y colectivos- por alcanzar una auténtica democratización de la cultura.

En estos años de triunfo neoliberal, reflexiona Colussi (2005), la información y la comunicación se han convertido en el más poderoso medio de sujeción de las poblaciones por parte de las elites: "la producción cultural actual, en vez de ser liberadora de la humanidad, dentro de los parámetros con que viene desplegándose en forma creciente no sólo es un fabuloso negocio monopolizado, sino que se ha transformado en una poderosa arma de control social uniformando sociedades e imponiendo un discurso único, favorable obviamente a los cada vez más reconcentrados grupos de poder".

En un sentido similar, Anderson (1999) sostiene que "el alto grado de concentración de la propiedad de los medios, en manos de las elites económicas y políticas, han clausurado hasta ahora las opciones de un desarrollo más democrático", lo que revela la dificultad de las clases hegemónicas regionales "para articular un modelo de acumulación que integre al conjunto de las sociedades".

Tal y como lo advierten la Relatoría de la Libertad de Expresión de la OEA y la UNESCO, e incluso algunos manifiestos de la sociedad civil durante la Cumbre de la Sociedad de la Información, efectuada en Ginebra en el 2003, la concentración es un fenómeno que amenaza el 
derecho de los individuos y los pueblos a promover, proteger su identidad y buscar caminos propios para su desarrollo cultural.

En este punto, Sánchez Ruiz (2006) establece una relación directa entre la posibilidad de hacer uso y de tener acceso a una pluralidad de fuentes de información y comunicación, y la expresión de las formas de vida y manifestaciones de creatividad "con las que los seres humanos producimos sentido, significamos el mundo, lo entendemos y lo proyectamos para las generaciones venideras".

Las limitaciones a la expresión de la diversidad, asociadas a la dependencia de los países latinoamericanos de los productos de la cultura de masas provenientes de Estados Unidos, y en menor medida de Europa, y de los flujos de intercambio financiero y comunicacional globales, están provocando problemas de invisibilidad social y exclusión en nuestras sociedades. En general, los grupos más vulnerables, como las mujeres, los pueblos indígenas (más de 40 millones de personas que casi no disponen de periódicos ni televisoras que produzcan contenidos en sus idiomas), los afrolatinoamericanos y las minorías, ocupan un lugar marginal y subordinado a prejuicios culturales, de honda raí: histórica.

Según Sánchez Ruiz (2006), algo similar ocurre "en términos de la función referencial de los medios, es decir, las v isibilidades de quienes se presentan (en los géneros informativos), o quienes se representan (en los géneros de ficción) a través de los mensajes mediáticos".

Distintas investigaciones realizadas a nivel mundial (incluyendo muestras latinoamericanas) para determinar quién figura en las noticias, confirman que "las mujeres siguen siendo el género sexual con menor visibilidad, a pesar de claros avances logrados durante los últimos decenios (...), tienden a aparecer en un segundo plano, además de que la 
propia desigualdad de género no se considera de interés noticioso. Igual pasa con etnias/razas, movimientos sociales, profesiones, y otras categorías sociales" (Sánchez Ruiz, 2006).

El sistema de medios de comunicación concentrados en América Latina, con sus mecanismos de organización simbólica de las relaciones sociales, parece constreñir las posibilidades de participación ciudadana efectiva y las garantías de que los puntos de vista de todos los sectores y grupos puedan hacerse oír en la sociedad, bajo una premisa que refleja el autoritarismo de la estructura oligopólica: "Lo que 'existe' en los medios no es tan diverso como el mundo real" (Sánchez Ruiz, 2006).

\section{La democracia mediática: una democracia limitada}

En condiciones ideales, los medios de comunicación posibilitarían la participación informada de los ciudadanos y ciudadanas, con igualdad de derechos, en cada uno de los supuestos que dan sustento a un sistema democrático: el conocimiento de las normas, el control sobre la arbitrariedad, la libre difusión de las ideas y de información alternativa, el sufragio, la publicidad de los actos administrativos, entre otros.

Sin embargo, este vínculo no se traduce, necesariamente, en consecuencias positivas per se, y mucho menos cuando la propiedad de los medios se concentra en cuatro grupos empresariales hegemónicos. Como explica Spellegrini (1991), el aporte de los medios a la vida democrática dependerá de cuánto contribuyan "efectivamente al equilibrio y distribución del poder. De otro modo, pueden destruir la democracia o al menos significativamente no contribuir a ella".

Esta autora señala que la acción polarizada o ideologizada de algunos medios puede llevar a que la sociedad se 
disuelva en compartimentos estancos, que no se interrelacionan ni siquiera para iniciar una discusión entre sus respectivas proposiciones. Un riesgo que es particularmente alto en aquellos países con regímenes democráticos que no están consolidados, y donde "el poder político convierte a los medios en simples extensiones de su función, o voceros de sus propios postulados" (Spellegrini, 1991).

En el contexto y complejidad de la globalización neoliberal, las sociedades latinoamericanas no han logrado establecer, en su praxis de la democracia, más allá de las formas y convenciones, una separación absoluta entre quienes representan los poderes institucionales y los poderes fácticos. Así, según lo describe Navarro Zamora (2005), "el poder real suele residir en instituciones a las que las normas asignan otras funciones o en grupos que no forman parte del orden político-institucional (familias tradicionales, grupos económicos y otros). Ante este divorcio se presenta un desconocimiento a los poderes decretados y en muchas ocasiones un descontento absoluto a los reales. En estos poderes reales hemos ubicado al poder mediático".

Este poder mediático emerge y se consolida a partir del diálogo entre el poder político y el poder económico (no siempre en ejercicio formal de la autoridad, valga decirlo) que, en criterio del sociólogo español Alberto Moncada (citado por Guinsburg, 2001), están implicados "corporativamente en que la democracia siga siendo manipulada desde arriba".

De tal suerte, la relación simbiótica entre medios de comunicación y poder político-económico en América Latina, conduce a lo que Guinsburg (2001) llama la democracia mediática que, a partir de la concentración de la propiedad de los medios y el manejo de la pauta publicitaria, opera en función de los negocios de los sectores vinculados a los poderes fácticos. Navarro Zamora (2005) considera que esta 
alianza les otorga a los empresarios de la comunicación "gran capacidad de generar opinión, determinar temas de agenda e incidir sobre la imagen pública de los funcionarios, partidos políticos e instituciones".

Una consecuencia inmediata de este asalto al poder institucional por parte de algunos medios y grupos con pretensiones hegemónicas, es que los ciudadanos y ciudadanas no se vinculan a un proceso pleno y efectivo de discusión pública; al mismo tiempo, los representantes políticos en los parlamentos asisten a la discusión de temas que ya han sido resueltos previamente a nivel mediático. Spellegrini (1991) identifica aquí una grave amenaza, porque "esta opinión pública simulada puede asemejarse más a una especie de absolutismo ilustrado que a un estado de derecho social y democrático".

Un ejemplo paradigmático permite ilustrar y contextualizar esta problemática. Se trata de la forma en que los cinco principales canales de televisión privados de Venezuela (Venevisión, Radio Caracas Televisión, Globovisión, Televen y CMT) y nueve de los diez grandes diarios nacionales (entre ellos El Universal, El Nacional, Tal Cual, El Impulso, El Nuevo País y El Mundo), se involucraron en el golpe de Estado contra el Presidente Hugo Chávez, el 11 de abril de 2002, prestando sus servicios a la difusión de información sesgada o manipulada.

Luis Bilbao (2002), periodista de Le Monde Diplomatique, considera que "los medios de comunicación en Venezuela dejaron de reflejar e interpretar los acontecimientos para pasar a diseñarlos según su voluntad, imponerlos como realidad virtual y luego conducirlos. La osada operación ha fallado. Pero deja hondas y peligrosas heridas en la sociedad venezolana e inaugura una fase singular de la lucha política, más allá de aquel país y del presidente Hugo Chávez (...)". 


\section{En los linderos del latifundio, se gestan las alternativas}

En su comentario al Informe Macbride, Gabriel García Márquez, miembro de la Comisión Internacional sobre Problemas de la Comunicación, dijo: "Unas estructuras más democráticas de comunicación constituyen una exigencia nacional e internacional para los pueblos de todo el mundo (...). La quiebra del poder concentrado en las manos de los intereses comerciales o burocráticos es un imperativo universal, y reviste una importancia especialmente crucial para los países del Tercer Mundo" (UNESCO, 1980).

¿Cómo avanzar hacia este objetivo? El periodista uruguayo Aram Aharonian (2006) señala una ruta: "comenzar a desalambrar los latifundios mediáticos latinoamericanos" (el resaltado no es del original).

La metáfora del desalambrado es más que una sugestiva construcción retórica: constituye una exigencia ética para posibilitar un desarrollo democrático, con justicia social, tolerancia, pluralidad y equidad en la región, en especial ante el vertiginoso avance de las nuevas tecnologías de la información y la comunicación en el contexto de la globalización neoliberal.

Las dos realidades del latifundio mediático han sido descritas por UNESCO (1995) como la convivencia de dos polos en América Latina: "en uno, la gran concentración de los medios que disponen de las tecnologías; en el otro, la proliferación de los medios regionales -que con sus limitaciones resultan propiciadores del pluralismo y la democratización- y de pequeños medios comunitarios, alternativos y populares".

Justamente es desde este último polo, ese que subsiste marginalmente en los linderos de la estructura oligopólica de la propiedad de los medios, y por encima de las abrumadoras diferencias materiales, donde se están gestando 
importantes transformaciones políticas y culturales en la esfera de la comunicación social.

Desde la sociedad civil, un vigoroso movimiento de radios comunitarias crece, especialmente, en el cono Sur del continente y en México; en la Internet, se multiplican los periódicos, revistas, radioemisoras alternativas en línea y otros foros interactivos, que articulan una amplia y representativa red de comunicación y resistencia entre los movimientos sociales latinoamericanos, "proporcionando voz a millones de personas que hasta ahora carecían de ella" (UNESCO, 1995).

Por su parte, algunos Estados recuperan, poco a poco, los espacios que fueron obligados a abandonar por la aplicación de las políticas del Consenso de Washington. Dos importantes y ambiciosos proyectos, que pretenden contribuir a la integración regional y al rescate de la memoria histórica, las tradiciones y la cultura latinoamericanas, ya se han puesto en marcha: la cadena TeleSUR, financiada por los gobiernos de Argentina, Cuba, Venezuela y Uruguay; y la Televisión del Sur, que promueve el gobierno de Lula da Silva en Brasil, para el fortalecimiento del MER. COSUR.

Del mismo modo, parece existir consenso en torno a la necesidad de establecer instrumentos legales que permitan controlar los procesos de concentración y fomenten el pluralismo y la diversidad de opiniones y culturas. Por ejemplo, la UNESCO (1980) y la OEA (2004) llaman la atención de sus Estados miembros para que adopten medidas jurídicas eficaces, congruentes con la doctrina de los Derechos Humanos, para limitar las prácticas monopólicas y oligopólicas en la propiedad de los medios; reducir la influencia de la publicidad sobre la política de redacción y los programas de radiodifusión; establecer directrices que planteen criterios de balance entre la eficiencia de los mercados 
de la comunicación y la pluralidad de la información; y, en definitiva, que estimulen el desarrollo de nuevos emprendimientos, de carácter ciudadano, independiente, o autónomo de los principales grupos productores y distribuidores de contenidos mediáticos.

Esta diversidad de propietarios y contenidos, consideran Mastrini y Becerra (2006) debe quedar reflejada en todos los niveles relevantes, para asegurar que "los medios de comunicación permitan la expresión del conjunto de las opiniones políticas y no sólo de aquellas afines a los intereses de los propietarios; (...) que las diferentes culturas presentes en un país o región encuentren un canal de comunicación [y reflejen] la diversidad, que es consustancial a toda sociedad moderna (...); [y para que] las minorías lingüísticas puedan expresarse y recibir información y programas en su lengua".

Las consideraciones expuestas adoptan, cada vez más, la forma de exigencias insoslayables para que la democracia no acabe simplemente en la emisión periódica del sufragio, sino que, por el contrario, asuma en las sociedades latinoamericanas su condición ideal de proceso continuo de ejercicio y repartición del poder, de modo de vida "que tiene que estar profundamente arraigada en los patrones culturales que se producen y reproducen en la vida cotidiana, en la familia, la escuela, el trabajo, los medios de difusión, y otros lugares de las esferas pública y privada" (Sánchez Ruiz, 1994).

En el reverso de la imagen de América Latina como "inmensa provincia del subdesarrollo", afirma Beneddetti (1987), hay también una historia "evidente e inconfundible: la que se escribe con hechos, con batallas, con trabajo, con dependencias y liberaciones". En el ámbito de las comunicaciones sociales, nuestros países tienen todavía una historia de liberación pendiente de escribir. Y sobre todo, de luchar. 


\section{Referencias bibliográficas}

AHARONIAN, A. 2006. Democratizar la información, ponencia presentada en la Cumbre del Movimiento de Países No Alineados, La Habana, 27 de agosto. Consultado el 5 de mayo de 2007, en: http://www.rebelion.org/ noticia.php?id $=36669$

ANDERSON, P. 1999. "Más allá del neoliberalismo: lecciones para la izquierda", en SADER, E. y GENTILI, P. (comps). La trama del neoliberalismo, Buenos Aires: CLACSO-Eudeba.

ARBILLA, D. 2006. "Para andar el camino", en: MASTRINI, G. y BECERRA, M. Periodistas y magnates. Estructura y concentración de la propiedad de las industrias culturales en América Latina. Buenos Aires: Instituto Prensa y Sociedad.

BENEDETTI, M. 1987. Subdesarrollo y letras de osadía. Madrid: Alianza Editorial.

BILBAO, L. 2002. "Dos semanas de fraude en Venezuela", en Le Monde Diplomatique, Edición Cono Sur, diciembre. Santiago de Chile.

CASTELLS, M. 2004. "La globalización truncada de América Latina", consultado el 25 de junio de 2005, en: http:// www.gobernabilidad.cl/modules.php?name= News\&file=print\&sid $=524$

COLUSSI, M. 2005. Del Informe McBride a Telesur, consultado: 23 de agosto de 2007, en: http://www.aporrea.org/actualidad/a16150.html

GUINSBURG, L. 2001. "El interés público", en Revista Encrucijadas, Universidad de Buenos Aires, año uno, nº 9.

HERMAN, E. y McCHESNEY, R. 1999. Los medios globales. Los nuevos misioneros del capitalismo corporativo. Madrid: Ediciones Cátedra.

KLIKSBERG, B. 2003. Hacia una economía con rostro humano. Asunción, Paraguay: Instituto de Capacitación y Estudios del Desarrollo. 
MASTRINI, G. y BECERRA, M. 2003. "50 años de concentración de medios en América Latina: del patriarcado artesanal a la valorización a escala", en Revista Digital Sala de Prensa, Edición agosto, n58, año V, vol. 2. Ver en: www.saladeprensa.org

MASTRINI, G. y BECERRA, M. 2006. Periodistas y magnates. Estructura y concentración de la propiedad de las industrias culturales en América Latina. Buenos Aires: Instituto Prensa y Sociedad.

MATTELART, A. 2003. "La información contra el Estado", en: RAMONET, I. ET AL. Nuevas tecnologias y concentración de los medios. La prensa, ¿refleja la realidad? Santiago de Chile: Editorial Aún creemos en los sueños, pp. 29-34.

MATTELART, A. 2006. "Por una nueva ecología de la comunicación", en: MASTRINI, G. y BECERRA, M. Periodistas y magnates. Estructura y concentración de la propiedad de las industrias culturales en América Latina. Buenos Aires: Instituto Prensa y Sociedad.

NAVARRO ZAMORA, L. 2005. "Democracia y medios, una disyuntiva en América Latina", en Estudios sobre el mensaje periodístico. Universidad Autónoma de San Luis Potosí. Consultado el 29 de marzo de 2007, En: http://www.ucm.es/ BUCM/revistas/inf/11341629/articulos/ ESMP0505110103A.PDF ().

OEA. 2004. Informe Anual de la Relatoría para la Libertad de Expresión. Washington: OAS.

RAMONET, I. 2001. La tiranía de la comunicación. Barcelona: Editorial Debate.

SÁNCHEZ RUIZ, E. 1994. "Los medios y la democracia en América Latina", en Revista Comunicación y Sociedad, núm. 20, abril. Centro de Estudios de la Información y la Comunicación, Universidad de Guadalajara.

2006. "Industrias culturales, diversidad y pluralismo en América Latina", en Cuadernos de Información y Comunicación, vol. 11. Universidad de Guadalajara.

SARAMAGO, J. 2003. Levantado del suelo. Madrid: Santillana Ediciones. 
SPELLEGRINI, S. 1991. Medios de comunicación, poder políico y democracia. Ponencia presentada en la reunión anual de ARCHI. Viña del Mar, 24 de julio. Facultad de Letras y Periodismo Universidad Católica, Santiago de Chile. Consultado el 17 de abril de 2007, en: http://fcom.altavoz.net/ prontus_fcom/site/artic/cuadernos/08/01_spellegrini.pdf

UNESCO, 1980. Un solo mundo, voces múltiples. Hacia un nuevo orden mundial más justo y eficaz de la Información y la Comunicación. México: Fondo de Cultura Económica.

UNESCO. 1995. Medios de comunicación y democracia en América Latina y el Caribe. Santiago de Chile: Ediciones UNES$\mathrm{CO}$.

URIBE, H. 2005. "Telesur: un paso de gigante contra la desinformación", consultado el 18 de marzo de 2006, en: http:// www.adital.org.br/site/noticia. asp?lang $=E S \&$ cod $=17845$ 